\title{
Analytic Formula for the Vibration and Sound Radiation of a Nonlinear Duct
}

\author{
Yiu-Yin Lee (iD \\ Department of Architecture and Civil Engineering, City University of Hong Kong, Kowloon Tong, Kowloon, Hong Kong \\ Correspondence should be addressed to Yiu-Yin Lee; bcraylee@cityu.edu.hk
}

Received 11 January 2019; Revised 27 March 2019; Accepted 14 April 2019; Published 28 April 2019

Academic Editor: Jean-Mathieu Mencik

Copyright ( 2019 Yiu-Yin Lee. This is an open access article distributed under the Creative Commons Attribution License, which permits unrestricted use, distribution, and reproduction in any medium, provided the original work is properly cited.

\begin{abstract}
This paper addresses the vibration and sound radiation of a nonlinear duct. Many related works assume that the boundaries are linearly vibrating (i.e., their vibration amplitudes are small), or that the duct panels are rigid, and their vibrations can thus be neglected. A classic method combined with Vieta's substitution technique is adopted to develop an analytic formula for computing the nonlinear structural and acoustic responses. The development of the analytic formula is based on the classical nonlinear thin plate theory and the three-dimensional wave equation. The main advantage of the analytic formula is that no nonlinear equation solver is required during the solution procedure. The results obtained from the proposed classic method show reasonable agreement with those from the total harmonic balance method. The effects of excitation magnitude, panel length, damping, and number of flexible panels on the sound and vibration responses are investigated.
\end{abstract}

\section{Introduction}

Over the past decades, many studies have considered structural-acoustic problems, plate vibration, and solution methods for nonlinear governing equations (e.g., [1-12]). Among various structural-acoustic problems, duct noise has been a particular focus for many years. For example, Venkatesham et al. [13] studied the breakout noise from a rectangular duct with compliant walls; they found that the low-frequency breakout noise was important and should not be neglected, and the coupling between the acoustic waves and the structural waves must be considered in the prediction of the transverse transmission loss. Tang and Lin [14] proposed the use of stiff light composite panels for duct noise reduction. Their selling point was that the actuation strategy would enable the creation of composite panels for duct noise control without the need for a traditional heavy structural mass. According to their results, the mass-spring resonance absorption in the case of a stiff thick panel with a thin flexible plate would be more efficient. Tiseo and Concilio [15] conducted a set of simulations for duct noise control. Their proposed feedback structural and acoustical structural control strategies maximized the damping within an acoustic duct and thus improved noise reduction. Jade and Venkatesham [16] implemented intensity-based experimental techniques for measuring breakout noise and validated "equivalent unfolded plate" analytical models. They developed an experimental test setup to measure the input and radiated sound power and vibration level and then calculated the transverse transmission loss and radiation efficiency. They adopted two measurement techniques: the intensity probe method (P-P method) and the microflown technique (P-U method). A numerical model was developed to predict transverse transmission loss and radiation efficiency, and the results were verified by the experimental results. In the aforementioned research works, nonlinear structural vibration, which should be considered for thin panel structures, was not the focus. The number of published articles on nonlinear structural acoustics is quite limited although a few are worth mentioning (e.g., [17-20]).

In contrast, there are numerous studies on solution methods for nonlinear governing equations (e.g., [21-28]). For example, Jacques et al. [29] adopted the harmonic balance and finite element methods for solving the problem of the nonlinear vibration of a viscoelastic sandwich beam. Chen et al. [30] analyzed the nonlinear steady state vibrations of plane structures. Both the finite element method and incremental harmonic balance 
method were used. The nonlinear governing equations were solved by the incremental harmonic balance method. Lee et al. [31] adopted the homotopy perturbation method to solve parabolic partial differential equations with constant coefficients for the nonlinear plate problem. Gao et al. [32] adopted the multiple scales method to analyze the nonlinear primary resonance of functionally graded porous cylindrical shells. Hao et al. [33] developed the nonlinear governing equations of motion for the FGM plates using Hamilton's principle and adopted the asymptotic perturbation method to obtain four-dimensional nonlinear averaged equations. When the aforementioned methods were applied to the nonlinear governing equations, coupled nonlinear algebraic equations were generated that needed to be solved by a nonlinear equation solver. This motivated the current study to adopt the classical method and Vieta's substitution technique [34] and to develop analytic formulations that would not require an equation solver to compute the nonlinear sound and vibration responses of a rectangular duct. Note that, normally a nonlinear problem would generate a set of nonlinear equations. A nonlinear equation solver is needed to solve them to obtain the nonlinear solutions (e.g. [35]). The effects of excitation magnitude, panel length, damping, and number of flexible panels on sound and vibration responses were also investigated for various panel cases.

Few studies have examined nonlinear structural acoustics, and studies of nonlinear duct sound and vibration are very limited. The modelling technique presented here is suitable for handling more than one flexible panel. Other works (e.g., [36, 37]) only considered one flexible panel. The main advantage of the solution method is that the procedure does not require a nonlinear equation solver; rather, the final nonlinear solutions can be expressed in terms of a set of symbolic parameters with various physical meanings. The method is very suitable for fast engineering calculation purposes. If a numerical method is used, the final solutions are numerical and require more computation time. The limitation of this solution method is that it can only be used for a simply supported plate. For other boundary conditions, the nonlinear modal differential equations are more complicated. Thus, no analytical solutions can be obtained from the proposed method.

\section{Theory and Formulation}

Figure 1 shows an open-end duct made of flexible panels. A piston-like excitation is generated at the other end. The acoustic pressure within the rectangular cavity can be obtained from the well-known homogeneous wave equation $[2,11,37]:$

$$
\left(\nabla^{2}+k^{2}\right) p_{Q}(x, y, z)=0
$$

where $p_{Q}(x, y, z)$ is the acoustic pressure at the position of $(x, y, z)$ induced by the $Q^{\text {th }}$ panel mode and $k$ is the wave number.

The solution form of the acoustic pressure and the acoustic cavity mode can be expressed in the following form:

$$
\begin{gathered}
P_{Q}(x, y, z)=\sum_{J=1}^{\bar{J}} P_{\mathrm{QJ}} \varphi_{J}(x, y, z), \\
\varphi_{J}(x, y, z)=\sin \frac{U \pi x}{2 a} \cos \frac{V \pi y}{b} \cos \frac{W \pi z}{c},
\end{gathered}
$$

where $P_{Q J}$ is the acoustic pressure amplitude of the $J^{\text {th }}$ acoustic mode; $J$ is the general acoustic mode number; $\bar{J}$ is the number of acoustic mode used; $U, V$, and $W$ are the acoustic mode numbers for the $x, y$, and $z$ directions; and $a$, $b$, and $c$ are the dimensions of the cavity. Note that $b$ and $c$ are much smaller than $a$. It is implied that the acoustic resonant frequencies of nonzero $V$ and $W$ modes are much higher than the first several acoustic resonant frequencies of the duct cavity.

Putting equations (2) and (3) into (1) and using the technique of integration by parts in [37] yield the following equation:

$$
\left(k^{2}-k_{J}^{2}\right) \alpha_{J J} P_{Q J}=-\int_{\text {pan }} \frac{\partial p_{Q}}{\partial n} \varphi_{J} d x d y,
$$

where $k_{J}=\sqrt{(U / 2 a)^{2}+(V / b)^{2}+(W / c)^{2}}$ is the wave number of the $J^{\text {th }}$ acoustic mode and $\alpha_{J J}=\int_{\text {vol }} \varphi_{J} \varphi_{J} d x d y d y$. The subscripts "vol" and "pan" represent the cavity volume and panel surface, respectively. $\partial p_{Q} / \partial n$ is the pressure gradient on the panel surface.

At the panel surface,

$$
\frac{\partial p_{\mathrm{Q}, h}}{\partial n}=\rho_{\mathrm{a}} \omega^{2} A_{\mathrm{Q}} \phi_{\mathrm{Q}}(x, y),
$$

where $\rho_{\mathrm{a}}$ is the air density; $\omega$ is the excitation frequency; $A_{o}$ and $A_{Q}$ are the displacement amplitudes of the source and panel, respectively; $\phi_{Q}(x, y)$ is the $Q^{\text {th }}$ panel mode shape, which is a double sine function, $\sin ((m \pi x) / a) \sin ((n \pi y) / b)$ (the panel is simply supported with immovable edges, and hence, the model shape can be written as a double sine function); and $m$ and $n$ are the structural mode numbers.

Putting equation (5) into (4) yields the following equation:

$$
P_{\mathrm{QJ}}=\frac{-\rho_{\mathrm{a}} \omega^{2}}{\left(k^{2}-k_{J}^{2}\right) \alpha_{J J}}\left(\alpha_{\mathrm{Q} J} A_{\mathrm{Q}}-\alpha_{o J} A_{o}\right),
$$

where $\alpha_{Q J}=\int_{\text {pan }} \phi_{Q} \varphi_{J} d x d y$ and $\alpha_{o J}=\int_{\text {sou }} \varphi_{J} d x d y$ and the subscript "sou" represents the vibration source.

In equation (6), the first term on the right side is the modal acoustic stiffness term, i.e.,

$$
\text { modal acoustic stiffness term, } K_{\mathrm{aco}, J}=\frac{\rho_{\mathrm{a}} \omega^{2} \alpha_{\mathrm{Q} J}}{\left(k^{2}-k_{J}^{2}\right) \alpha_{J J}} \text {. }
$$

If there are 2 or more flexible panels considered, the overall acoustic stiffness is given by

overall acoustic stiffness term,

$$
K_{\mathrm{aco}}=N \rho_{\mathrm{a}} \omega^{2} \sum_{J=1}^{\bar{J}} \frac{\alpha_{\mathrm{Q} J}}{\left(k^{2}-k_{J}^{2}\right) \alpha_{J J}} \frac{\alpha_{\mathrm{Q} J}}{\alpha_{\mathrm{QQ}}},
$$




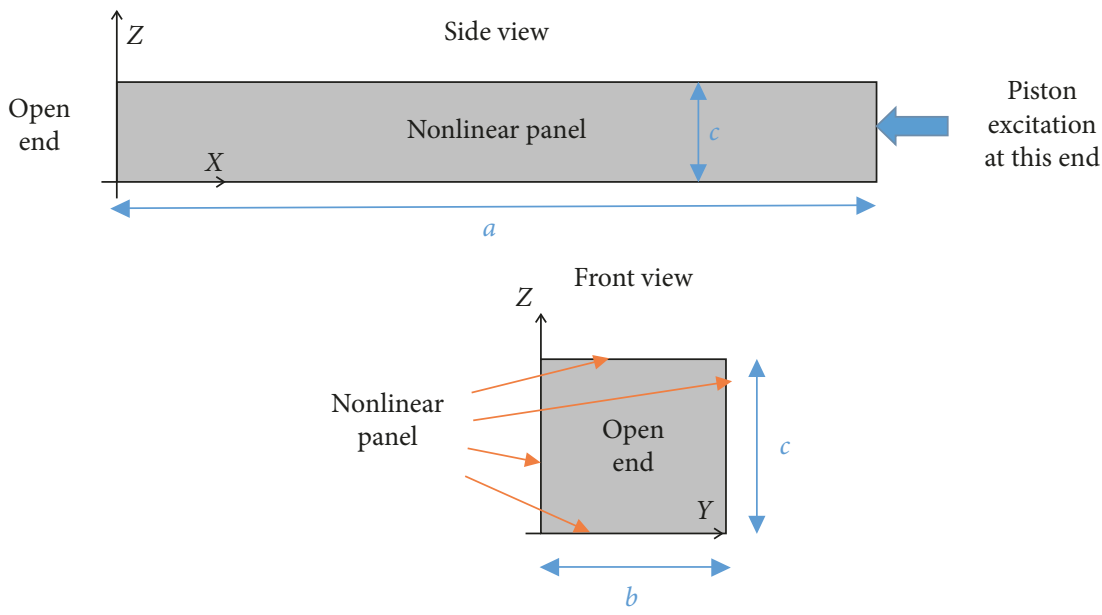

Figure 1: A duct/tube formed by nonlinear flexible panels.

where $N$ is the number of panels and the subscript "aco" means acoustics. It is noted that the term on the right side is the acoustic stiffness force term. Note that in equation (8), $\alpha_{\mathrm{QJ}} / \alpha_{\mathrm{QQ}}$ is the modal contribution factor and $\alpha_{\mathrm{QQ}}=$ $\int_{\mathrm{pan}} \phi_{\mathrm{Q}} \phi_{\mathrm{Q}} d x d y$.

According to [38, 39], the Airy stress function derivatives can be expressed as follows:

$$
\begin{aligned}
\frac{\partial^{2} \bar{F}}{\partial \xi^{2}} & =\frac{\partial \bar{v}}{\partial \eta}+\frac{1}{2}\left(\frac{\partial \bar{w}}{\partial \eta}\right)^{2}+\nu\left[\frac{\partial \bar{u}}{\partial \xi}+\frac{1}{2}\left(\frac{\partial \bar{w}}{\partial \xi}\right)^{2}\right], \\
\frac{\partial^{2} \bar{F}}{\partial \eta^{2}} & =\frac{\partial \bar{u}}{\partial \xi}+\frac{1}{2}\left(\frac{\partial \bar{w}}{\partial \xi}\right)^{2}+\nu\left[\frac{\partial \bar{v}}{\partial \eta}+\frac{1}{2}\left(\frac{\partial \bar{w}}{\partial \eta}\right)^{2}\right], \\
-\frac{\partial^{2} \bar{F}}{\partial \xi \partial \eta} & =\frac{1-v}{2}\left[\frac{\partial \bar{u}}{\partial \eta}+\frac{\partial \bar{v}}{\partial \xi}+\frac{\partial \bar{w}}{\partial \eta} \frac{\partial \bar{w}}{\partial \xi}\right] .
\end{aligned}
$$

The solution forms of $\bar{w}, \bar{u}$, and $\bar{v}$ are given in [40]:

$$
\begin{aligned}
& \bar{w}=\bar{\Lambda}_{Q} \sin (\pi \zeta) \sin (\pi r \eta), \\
& \bar{u}=\frac{\left(\bar{\Lambda}_{Q}\right)^{2} \pi}{16}\left(\cos (2 \pi r \eta)-1+v r^{2}\right) \sin (2 \pi \zeta), \\
& \bar{v}=\frac{\left(\bar{\Lambda}_{Q}\right)^{2} \pi}{16}\left(r \cos (2 \pi r \zeta)-r+\frac{\nu}{r}\right) \sin (2 \pi r \eta) .
\end{aligned}
$$

Hence, the governing equation of Airy stress function is given by

$$
\frac{1}{12}\left(\frac{d^{2} \bar{w}}{d \tau^{2}}+\nabla^{4} \bar{w}\right)=\left(\frac{\partial^{2} \bar{F}}{\partial \eta^{2}} \frac{\partial^{2} \bar{w}}{\partial \xi^{2}}+\frac{\partial^{2} \bar{F}}{\partial \xi^{2}} \frac{\partial^{2} \bar{w}}{\partial \eta^{2}}-2 \frac{\partial^{2} \bar{F}}{\partial \eta \partial \xi} \frac{\partial^{2} \bar{w}}{\partial \eta \partial \xi}\right),
$$

where $\bar{\Lambda}_{Q}=\left(A_{Q} / h\right) T(\tau), T(\tau)$ is a time function $\leq 1 ; h$ is the panel thickness; $\bar{F}$ is the Airy stress function; $\bar{w}, \bar{u}$, and $\bar{v}$ are the dimensionless displacements along the $z, x$, and $y$ directions, respectively; $v$ is Poisson's ratio; $\zeta=m x / a$ and $\eta=$ $n y / a$ are the dimensionless $x$ and $y$ coordinates; $r=a / b$ is the panel aspect ratio; $\tau=\left(C_{\mathrm{p}} t\right) /(\sqrt{12} a)$ is nondimensional time; $C_{\mathrm{p}}=\sqrt{E /\left(\rho_{\mathrm{p}}\left(1-\nu^{2}\right)\right)}$ is the phase velocity of compressional waves; $E$ is Young's modulus; $\rho_{\mathrm{p}}$ is the panel density; and $t$ is time.

Hence, the terms with $\bar{F}$ in equation (11) can be replaced by equations $(9 a)-(9 c)$. Then, according to [38], the higher mode terms (i.e., $\sin (3 \pi \zeta) \sin (\pi r \eta), \sin (\pi \zeta) \sin (3 \pi r \eta), \ldots)$ are neglected, and the nonlinear governing equation of free plate vibration is expressed in the following form:

$$
D_{1} \frac{d^{2} \bar{\Lambda}_{Q}}{d \tau^{2}}+D_{2} \bar{\Lambda}_{Q}+D_{3} \bar{\Lambda}_{Q}^{3}=0,
$$

where $D_{1}, D_{2}$, and $D_{3}$ are the constants in terms of $r, v, E, a$, $b, \ldots$ etc. The variables in the nonlinear governing equation (i.e., equations (12a)-(12c)) are dimensionless. Note that the above equation is for free vibration only (i.e., no force term). In this study, the governing equation of nonlinear forced vibration is dimensional and shown as follows.

The following dimensional variables are introduced:

$$
\begin{aligned}
\Lambda_{Q} & =h \bar{\Lambda}_{Q}, \\
t & =\frac{\sqrt{12} a}{C_{\mathrm{p}}} \tau .
\end{aligned}
$$

Then, put equations (12b) and (12c) into (12a) to eliminate the two nondimensional variables (i.e., $\bar{\Lambda}_{Q}$ and $\tau$ ). Hence, the governing equation of nonlinear free vibration is given by the following equation:

$$
\rho_{\mathrm{p}} \frac{d^{2} \Lambda_{\mathrm{Q}}}{d t^{2}}+\rho_{\mathrm{p}} \omega_{\mathrm{Q}}^{2} \Lambda_{\mathrm{Q}}+\beta_{\mathrm{Q}} \Lambda_{\mathrm{Q}}^{3}=0 .
$$

Consider the acoustic stiffness force term in equation (8) and a force term which represents uniform harmonic excitation. Equation (13a) can be rewritten as follows:

$$
\begin{aligned}
& \rho_{\mathrm{p}} \frac{d^{2} \Lambda_{\mathrm{Q}}}{d t^{2}}+\left[\rho_{\mathrm{p}} \omega_{\mathrm{Q}}^{2}+N \rho_{\mathrm{a}} \omega^{2} \sum_{J=1}^{\bar{J}} \frac{\alpha_{\mathrm{Q} J}}{\left(k^{2}-k_{J}^{2}\right) \alpha_{J J}} \frac{\alpha_{\mathrm{Q} J}}{\alpha_{\mathrm{QQ}}}\right] \Lambda_{\mathrm{Q}} \\
& +\beta_{\mathrm{Q}} \Lambda_{\mathrm{Q}}^{3}+\kappa \rho_{\mathrm{p}} g \frac{\alpha_{o J}}{\alpha_{\mathrm{QQ}}} \sin (\omega t)=0,
\end{aligned}
$$


where $\kappa=$ dimensionless excitation parameter, $g=9.81 \mathrm{~m} \cdot \mathrm{s}^{-2}$, $\Lambda_{Q}=A_{Q} T(t), \beta_{Q}=\left(E h / 4\left(1-v^{2}\right)\right)(m \pi / b)^{4}\left[\left(1+((n / m) r)^{4}\right)\right.$ $\left.\left((3 / 4)-\left(v^{2} / 4\right)\right)+v((n / m) r)^{2}\right]$, and note that again $\alpha_{\mathrm{QJ}} / \alpha_{\mathrm{QQ}}$ is the modal contribution factor, which is the same as the one in equation (8).

In equation (13b), the nonlinearity of the structuralacoustic system is represented by the term $\beta_{Q} \Lambda_{Q}^{3}$. Physically, the nonlinearity is caused by the large amplitude vibration. In a typical linear vibration (or small vibration) analysis, the axial and transverse vibrations of a panel are independent, whereas they are coupled in a nonlinear vibration analysis. They are coupled in the nonlinear analysis because in small deflection cases, it is assumed that the direction of the panel's transverse vibration is still perpendicular to the surface; in large deflection cases, the transverse vibration direction is no longer perpendicular to the surface because the surface is subject to large deformation. Mathematically, the nonlinear term in equations (13a) and (13b) results in multiple solutions, whereas there is only one solution in linear cases. In fact, equations (13a) and (13b) are in the form of the well-known Duffing equation.

Consider the approximation of $T(t) \approx \sin (\omega t)$, neglect the higher harmonic terms, and then equation (13b) is rewritten in the following form:

$$
\frac{3}{4} \beta_{Q} A_{Q}^{3}+\Pi_{Q} A_{Q}+\Gamma_{Q}=0
$$

where $\Gamma_{\mathrm{Q}}=\kappa \rho_{\mathrm{p}} g\left(\alpha_{\mathrm{QJ}} / \alpha_{\mathrm{QQ}}\right)$ and $\Pi_{\mathrm{Q}}=\rho_{\mathrm{p}}\left(\omega_{\mathrm{Q}}^{2}-\omega^{2}\right)+N \rho_{\mathrm{a}} \omega^{2}$ $\sum_{J=1}^{\bar{J}}\left(\alpha_{\mathrm{QJ}} /\left(\left(k^{2}-k_{J}^{2}\right) \alpha_{J J}\right)\right)\left(\alpha_{\mathrm{Q} J} / \alpha_{\mathrm{QQ}}\right) . \omega_{\mathrm{Q}}$ is the resonant frequency of the $Q^{\text {th }}$ panel mode.

Note that there is a limitation in the proposed solution form. In highly nonlinear cases, superharmonic and subharmonic responses occur. As the solution form does not include the components of $\sin (3 \omega t)$ or $\sin (1 / 3 \omega t)$, the results cannot show these phenomena.

For damped vibration, $A_{Q}$ in equation (14) is rewritten into the following complex form:

$$
\begin{aligned}
\left|A_{Q}\right| & =\frac{\left|\Gamma_{Q}\right|}{\left.\left|\Pi_{Q}+(3 / 4) \beta_{Q}\right| A_{Q}\right|^{2} \mid}, \\
\Longrightarrow\left|A_{Q}\right|^{2} & =\frac{\left|\Gamma_{Q}\right|^{2}}{\left(\operatorname{Re}\left\langle\Pi_{Q}\right\rangle+(3 / 4) \beta_{Q}\left|A_{Q}\right|^{2}\right)^{2}+\left(\operatorname{Im}\left\langle\Pi_{Q}\right\rangle\right)^{2}} .
\end{aligned}
$$

Then, substituting equation (16) in (14), we get the following equation:

$$
G_{3} \gamma^{3}+G_{2} \gamma^{2}+G_{1} \gamma+G_{0}=0
$$

where $\gamma=\left|A_{Q}\right|^{2} ; \quad G_{3}=\left((3 / 4) \beta_{Q}\right)^{2} ; G_{2}=(3 / 2) \beta_{Q} \operatorname{Re}\left\langle\Pi_{Q}\right\rangle$; $G_{1}=\left|\Pi_{Q}\right|^{2} ; G_{0}=\left|\Gamma_{Q}\right|^{2} ; \operatorname{Re}\left\langle\Pi_{Q}\right\rangle$ is the real part of $\Pi_{Q}$; $\operatorname{Im}\left\langle\Pi_{Q}\right\rangle$ is the imaginary part of $\Pi_{Q} ; \Pi_{Q}=\rho_{\mathrm{p}}\left(\omega_{Q}^{2}-\omega^{2}\right)+$ $i\left(2 \zeta \omega \omega_{\mathrm{Q}}\right)+N \rho_{\mathrm{a}} \omega^{2} \sum_{J=1}^{\bar{J}}\left(\alpha_{\mathrm{Q} J} /\left(\left(k^{2}+i\left(2 \zeta k k_{J}\right)-k_{J}^{2}\right) \alpha_{J J}\right)\right)\left(\alpha_{\mathrm{Q} J} /\right.$ $\left.\alpha_{\mathrm{QQ}}\right) ; i$ is equal to $\sqrt{-1}$; and $\zeta$ is the damping ratio. Physically, the damping force is assumed to be proportional to the damping ratio $\times$ the velocity (i.e., $d \Lambda_{Q} / d t$ ). The damping ratio represents the level of damping relative to the critical damping (i.e., $\zeta=1$ ).

According to Vieta's substitution [34], equation (17) can be simplified using the substitution of $\gamma=\lambda-\left(G_{2} / 3 G_{3}\right)$ :

$$
\lambda^{3}+\varepsilon_{1} \lambda+\varepsilon_{0}=0
$$

where $\varepsilon_{1}=\left(3 G_{3} G_{1}-G_{2}^{2}\right) / 3 G_{3}^{2}$ and $\varepsilon_{0}=\left(2 G_{2}^{3}-9 G_{3} G_{2} G_{1}+\right.$ $\left.27 G_{3}^{2} G_{0}\right) / 27 G_{3}^{3}$.

Equation (18) can be further simplified using the substitutions of $\lambda=B-\left(\varepsilon_{1} / 3 B\right)$ and $\chi=B^{3}$ :

$$
\chi^{2}+\varepsilon_{0} \chi-\frac{\varepsilon_{1}^{3}}{27}=0
$$

The exact solutions of equation (19) are easily obtainable without any nonlinear equation solver. Finally, the solutions of $\lambda$ are given below:

$$
\begin{aligned}
& \lambda_{1}=-\frac{1}{3} \sqrt[3]{\frac{1}{2}\left(\delta_{1}+\delta_{2}\right)}-\frac{1}{3} \sqrt[3]{\frac{1}{2}\left(\delta_{1}-\delta_{2}\right)} \\
& \lambda_{2}=\frac{1+i \sqrt{3}}{6} \sqrt[3]{\frac{1}{2}\left(\delta_{1}+\delta_{2}\right)}+\frac{1-i \sqrt{3}}{6} \sqrt[3]{\frac{1}{2}\left(\delta_{1}-\delta_{2}\right)}, \\
& \lambda_{3}=\frac{1-i \sqrt{3}}{6} \sqrt[3]{\frac{1}{2}\left(\delta_{1}+\delta_{2}\right)}+\frac{1+i \sqrt{3}}{6} \sqrt[3]{\frac{1}{2}\left(\delta_{1}-\delta_{2}\right)},
\end{aligned}
$$

where $\delta_{1}=\sqrt{\left(27 \varepsilon_{1}\right)^{2}+4\left(3 \varepsilon_{0}\right)^{3}}$ and $\delta_{2}=27 \varepsilon_{1}$.

Hence, $\left|A_{Q}\right|$ can be found from $\gamma=\lambda-\left(G_{2} / 3 G_{3}\right)$ and $\gamma=\left|A_{Q}\right|^{2}$. Then, for the calculation of sound radiation, the following equations can be used to find the radiation efficiency [41]:

$$
\begin{aligned}
\sigma_{Q}= & \frac{32 k^{2} a b}{m^{2} n^{2} \pi^{5}}\left\{1-\frac{k^{2} a b}{12}\left[\left(1-\frac{8}{(m \pi)^{2}}\right) \frac{a}{b}\right.\right. \\
& \left.\left.+\left(1-\frac{8}{(n \pi)^{2}}\right) \frac{b}{a}\right]\right\}, \quad \text { for symmetric modes, } \\
\sigma_{Q}= & \frac{8 k^{4} a^{3} b}{3 m^{2} n^{2} \pi^{5}}\left\{1-\frac{k^{2} a b}{20}\left[\left(1-\frac{24}{(m \pi)^{2}}\right) \frac{a}{b}\right.\right. \\
& \left.\left.+\left(1-\frac{8}{(n \pi)^{2}}\right) \frac{b}{a}\right]\right\}, \quad \text { for antisymmetric modes, }
\end{aligned}
$$

where symmetric modes are the modes of $m$ and $n$ set to odd numbers and antisymmetric modes are the modes of $m$ and/ or $n$ set to even numbers.

\section{Numerical Results and Discussion}

The material and physical properties adopted in the following numerical cases are as follows: Young's modulus = $7.1 \times 10^{10} \mathrm{~N} / \mathrm{m}^{2}$; Poisson's ratio $=0.3$; panel thickness $=$ $2 \mathrm{~mm}$; panel density $=2700 \mathrm{~kg} / \mathrm{m}^{3}$; air density $=1.2 \mathrm{~kg} / \mathrm{m}^{3}$; sound speed $=340 \mathrm{~m} / \mathrm{s}$; and cross-sectional dimensions of the duct $=0.5 \mathrm{~m} \times 0.5 \mathrm{~m}$. Tables $1-3$ show the acoustic mode convergence studies for various excitation levels. The 
damping ratio, $\zeta$, is 0.02 . The seven mode solutions are normalized as 100 . In the $(1,1)$ and $(2,1)$ mode cases, the four acoustic mode solutions achieve an error rate of less than $1 \%$ for different excitation levels ( $\kappa=0.05$ to 0.5 ), while in the $(3,1)$ mode case, the six mode solutions achieve an error rate of around 3.5\%. Tables 4-6 show the structural mode contributions for various excitation levels: the larger the excitation level is, the higher the contributions of the two higher modes are. In other words, when the excitation is high, more modes are needed. The comparisons between Tables 4-6 show the modal contributions at the corresponding peak frequencies. The contributions of the dominant modes at the corresponding peak frequencies are always higher than $94 \%$. Thus, the use of two structural modes is appropriate for obtaining solutions with good accuracy. Figures 2(a) and 2(b) present the comparisons between the first symmetric and first antisymmetric modal amplitude solutions obtained from the proposed method and the classical harmonic balance method (e.g., [20]). The nondimensional excitation parameter value, $\kappa$, is 0.5 . The damping ratio, $\zeta$, is 0.02 . The panel length $a$ is $3 \mathrm{~m}$, and the number of flexible panels is four. Two harmonic components, $\sin (\omega t)$ and $\sin (3 \omega t)$, are adopted in the classical harmonic balance method. The results obtained from the two methods are generally in good agreement for the first symmetric and first antisymmetric modal amplitude solutions. The only slight differences are found at the first nonlinear peak values, around the frequencies $\omega / \omega_{1}=0.9$ in Figure 2(a) and 1.15 in Figure 2(b). The differences are caused by the different definitions of the damping terms in the two methods. There is a limitation in the proposed solution form. In highly nonlinear cases, superharmonic and subharmonic responses occur. As the solution form does not include the $\sin (3 \omega t)$ or $\sin (1 / 3 \omega t)$ components, the results cannot show these phenomena.

Figures 3(a)-3(d) show the symmetric and antisymmetric vibration amplitudes and sound powers plotted against the excitation frequency for various excitation magnitudes. The damping ratio $\zeta$ is 0.02 ; the first two structural modes and seven acoustic modes are adopted; four flexible panels are considered $(N=4)$; and the panel length $a=3 \mathrm{~m}$. The peak frequencies and peak values of the structural resonances (i.e., the first two peaks in the figures) increase with the excitation magnitude. The peak values of the other resonances (i.e., acoustic resonances) also increase with the excitation magnitude. The peak frequencies remain almost unchanged, and the peaks are generally more linear, except those under the high excitation level $\kappa=0.5$, which are more nonlinear, and their peak frequencies are around $\omega / \omega_{1}=2.3$. The comparisons between the vibration amplitude and sound power curves show that the high frequency acoustic peaks are more important in the sound power figures while the low frequency structural peaks are more important in the vibration amplitude figures because the radiation efficiencies are monotonically increasing with the excitation frequency (Figure 3(e)). The higher the excitation frequency, the higher the radiation efficiency. The comparisons between the curves of symmetric and antisymmetric cases show that the second or higher acoustic peaks
TABLE 1: $(1,1)$ mode peak vibration amplitude convergence for various excitation magnitudes ( 3 symmetric structural modes, $\zeta=0.02)$.

\begin{tabular}{lccc}
\hline No. of acoustic modes & $\kappa=0.05$ & 0.2 & 0.5 \\
\hline 1 & 78.7 & 82.4 & 84.7 \\
4 & 99.9 & 99.9 & 99.7 \\
6 & 100.0 & 100.0 & 100.0 \\
7 & 100.0 & 100.0 & 100.0 \\
\hline
\end{tabular}

TABle 2: $(2,1)$ mode peak vibration amplitude convergence for various excitation magnitudes ( 3 antisymmetric structural modes, $\zeta=0.02)$.

\begin{tabular}{lccc}
\hline No. of acoustic modes & $\kappa=0.05$ & 0.2 & 0.5 \\
\hline 1 & 62.5 & 69.5 & 75.2 \\
4 & 100.1 & 100.0 & 99.9 \\
6 & 99.8 & 99.8 & 99.6 \\
7 & 100.0 & 100.0 & 100.0 \\
\hline
\end{tabular}

TABle 3: $(3,1)$ mode peak vibration amplitude convergence for various excitation magnitudes (3 symmetric structural modes, $\zeta=0.02)$.

\begin{tabular}{lccc}
\hline No. of acoustic modes & $\kappa=0.05$ & 0.2 & 0.5 \\
\hline 1 & 66.4 & 70.3 & 76.2 \\
4 & 67.5 & 75.2 & 80.2 \\
6 & 101.2 & 96.2 & 96.9 \\
7 & 100.0 & 100.0 & 100.0 \\
\hline
\end{tabular}

TABle 4: Mode contribution at the $(1,1)$ mode peak frequency $(\kappa=0.2, \zeta=0.02)$.

\begin{tabular}{lccc}
\hline No. of acoustic modes & $(1,1)$ mode & $(3,1)$ mode & $(5,1)$ mode \\
\hline 1 & 95.6 & 3.1 & 1.2 \\
4 & 96.2 & 2.7 & 1.1 \\
6 & 95.8 & 3.1 & 1.1 \\
7 & 95.8 & 3.1 & 1.1 \\
\hline
\end{tabular}

TABle 5: Mode contribution at the $(2,1)$ mode peak frequency $(\kappa=0.2, \zeta=0.02)$.

\begin{tabular}{lccc}
\hline No. of acoustic modes & $(2,1)$ mode & $(4,1)$ mode & $(6,1)$ mode \\
\hline 1 & 93.6 & 5.1 & 1.3 \\
4 & 94.9 & 4.0 & 1.0 \\
6 & 95.0 & 4.0 & 1.0 \\
7 & 94.7 & 4.2 & 1.0 \\
\hline
\end{tabular}

TABLE 6: Mode contribution at the $(3,1)$ mode peak frequency $(\kappa=0.2, \zeta=0.02)$.

\begin{tabular}{lccc}
\hline No. of acoustic modes & $(1,1)$ mode & $(3,1)$ mode & $(5,1)$ mode \\
\hline 1 & 0.3 & 95.6 & 4.1 \\
4 & 0.2 & 95.8 & 4.0 \\
6 & 0.2 & 96.3 & 3.5 \\
7 & 0.2 & 96.4 & 3.4 \\
\hline
\end{tabular}




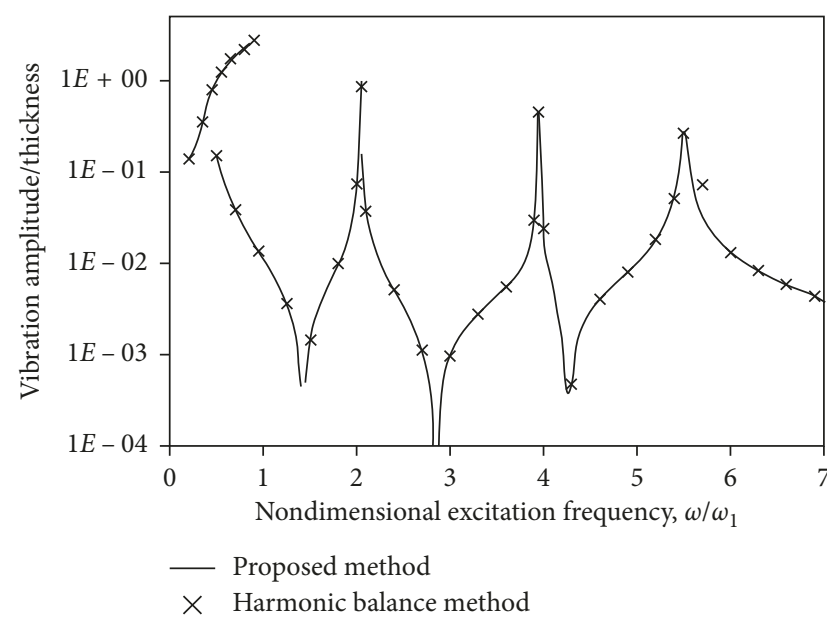

(a)

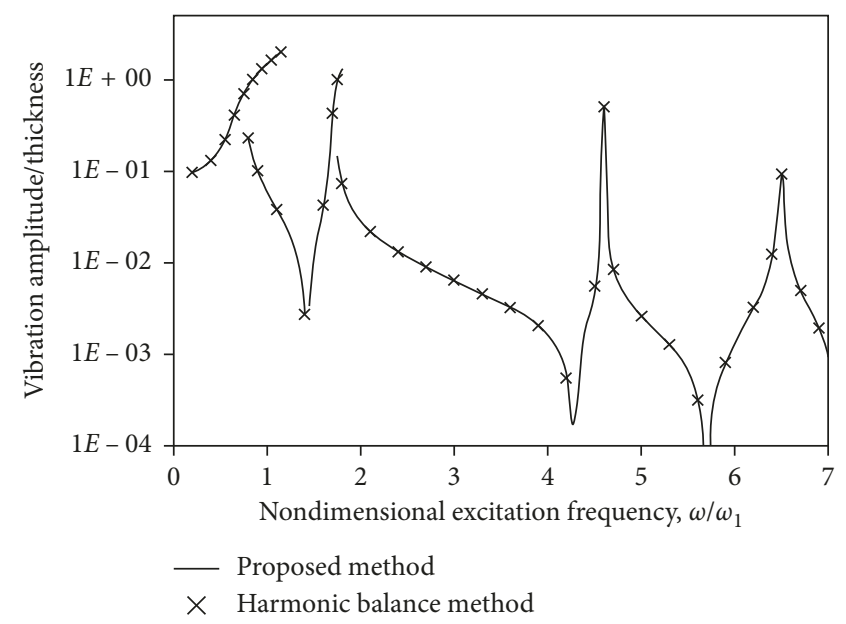

(b)

Figure 2: (a) Comparison of the symmetric and (b) antisymmetric vibration amplitude results from the proposed method and classical harmonic balance method $(\kappa=0.5, a=3 \mathrm{~m}, b=c=0.5 \mathrm{~m}, \xi=0.02$, and $N=4)$.

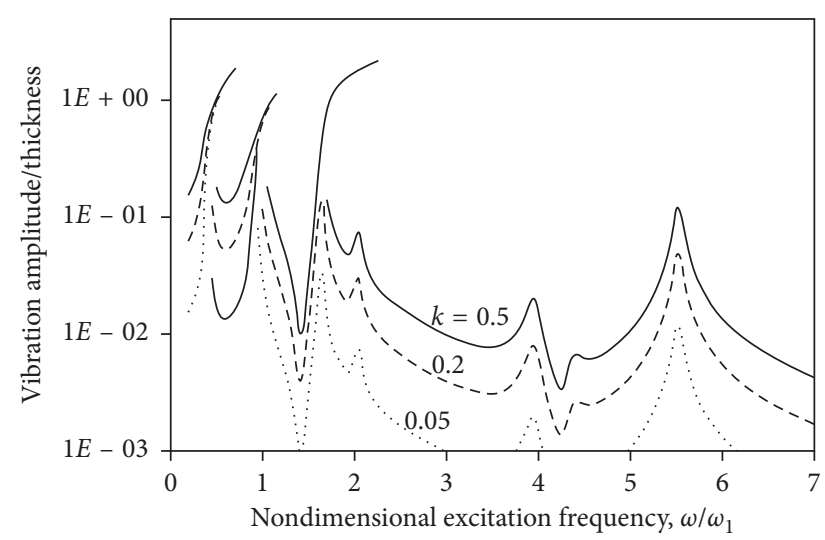

(a)

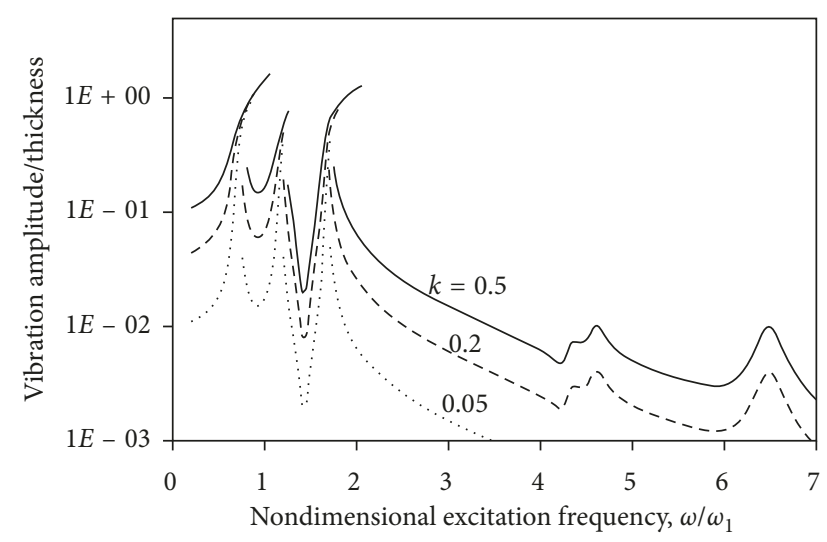

(c)

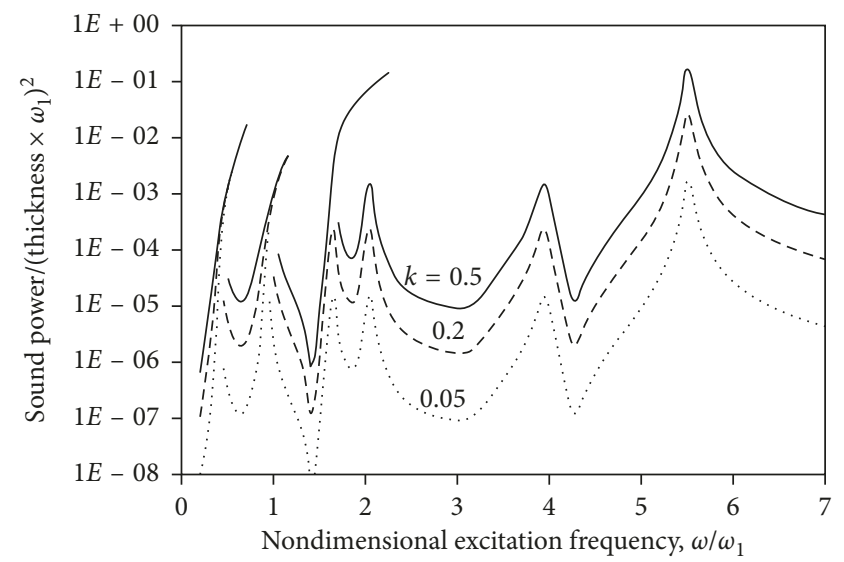

(b)

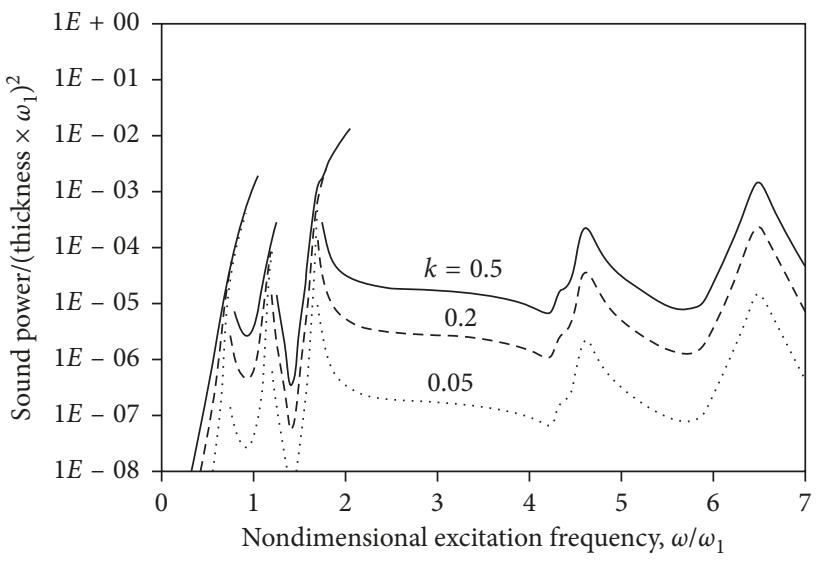

(d)

Figure 3: Continued. 


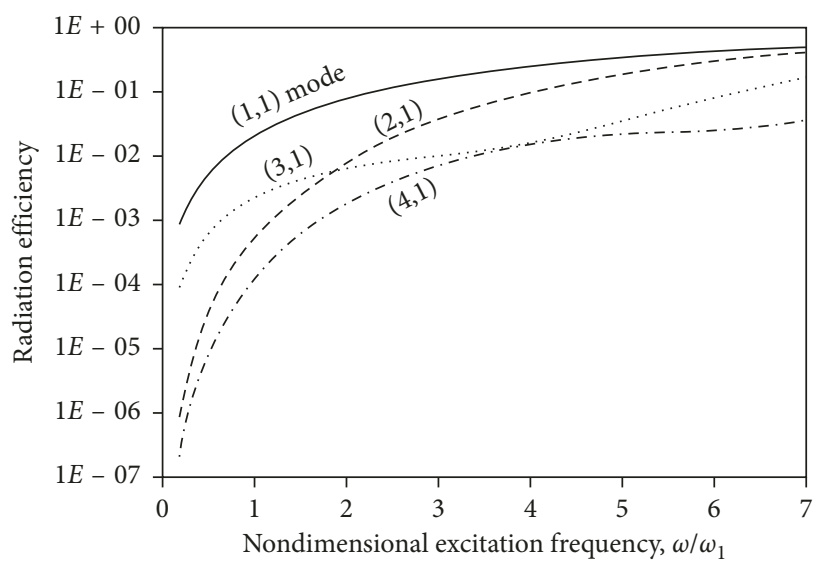

(e)

FIGURE 3: (a) Symmetric and (c) antisymmetric vibration amplitude versus excitation frequency for various source excitation magnitudes ( $a=3 \mathrm{~m}, b=c=0.5 \mathrm{~m}, \xi=0.02$, and $N=4$ ); (b) symmetric and (d) antisymmetric sound radiation versus excitation frequency for various source excitation magnitudes $(a=3 \mathrm{~m}, b=c=0.5 \mathrm{~m}, \xi=0.02$, and $N=4)$; (e) radiation efficiency versus excitation frequency for various structural modes.

are much less significant in the antisymmetric case, while the first acoustic peaks are important in both the symmetric and antisymmetric cases.

Figures 4(a)-4(d) show the symmetric and antisymmetric vibration amplitudes and sound powers plotted against the excitation frequencies for various damping ratios. The excitation level $\kappa=0.2$; the first two structural modes and seven acoustic modes are adopted; four flexible panels are considered $(N=4)$; and the panel length $a=3 \mathrm{~m}$. Obviously, the damping only affects the peak and trough values. In the off-resonance range, the damping has no significant effect. If the damping ratio is small $(\zeta=0.005)$, the two structural peaks and first acoustic peaks look more nonlinear, the peak values are the highest among the three damping cases, and the trough values (or antiresonant values) are the lowest. If the damping ratio is high $(\zeta=0.05)$, (1) the two structural peaks and first acoustic peaks, which look more nonlinear in the case of $\zeta=0.005$, become more linear; and (2) the peak values are the lowest and the trough values the highest. Figures 5(a)-5(d) show the symmetric and antisymmetric vibration amplitudes and sound powers plotted against the excitation frequencies for various panel lengths. The excitation level $\kappa=0.2$; the damping ratio $\zeta=0.02$; the first two structural modes and seven acoustic modes are adopted; and four flexible panels are considered $(N=4)$. Obviously, the panel length (or cavity length) affects the structural and acoustic resonant frequencies in all vibration amplitude and sound power figures. Generally, the shorter the panel length is, the higher the resonant frequencies and peak values are. Note that, in the case of $a=2$, some acoustic peaks are outside the frequency range. In Figures 5(c) and 5(d), the resonant frequencies of the acoustic peaks around $\omega / \omega_{1}=6.4$ to 6.6 are not significantly affected by the panel length. The first antisymmetric acoustic peaks are more nonlinear than the first symmetric acoustic peaks. This phenomenon can also be observed in Figures 6(c) and 6(d).
Figures 6(a)-6(d) show the symmetric and antisymmetric vibration amplitudes and sound powers plotted against the excitation frequencies for various numbers of flexible panels. The excitation level $\kappa=0.2$; the damping ratio $\zeta=0.02$; the first two structural modes and seven acoustic modes are adopted; and the panel length $a=3 \mathrm{~m}$. Figures 6(a) and 6(c) show that the number of panels affects the peak values, peak frequencies, and off-resonance responses in all cases. If only one flexible panel is installed, (1) the first and second symmetric and antisymmetric structural resonant frequencies among the three cases (i.e., $N=1,2,4)$ are highest in both the vibration amplitude and the sound power figures; (2) the first symmetric and antisymmetric structural resonant peak values in the sound power figures are also the highest, while the first symmetric and antisymmetric structural resonant peak values in the vibration amplitude figures are nearly the same among the three cases; (3) the second symmetric and antisymmetric structural resonant peak values in the vibration amplitude and sound power figures are the lowest among the three cases; and (4) the first antisymmetric acoustic peaks are more nonlinear (the jump phenomenon can be seen), while the second antisymmetric structural peaks look more linear.

Figures $7(\mathrm{a})-7(\mathrm{~d})$ show the structural-acoustic peak ratios plotted against the dimensionless excitation level for various structural modes. The damping ratio $\zeta=0.02$; the first seven acoustic modes are adopted; four flexible panels are considered $(N=4)$; and the panel length $a=2 \mathrm{~m}$. The structural-acoustic peak ratio is defined as the ratio of the structural peak value to the highest acoustic peak value. In Figure $7(\mathrm{a})$, the two structural-acoustic peak ratios are monotonically decreasing with the increasing excitation level. This implies that the acoustic peak is more important or significant when the excitation level is higher. In Figures 7 (b) and $7(\mathrm{c})$, the structural-acoustic peak ratios of the vibration amplitude are almost constant when the excitation level ranges from $\kappa=0.05$ to 0.12 . There is an abrupt 


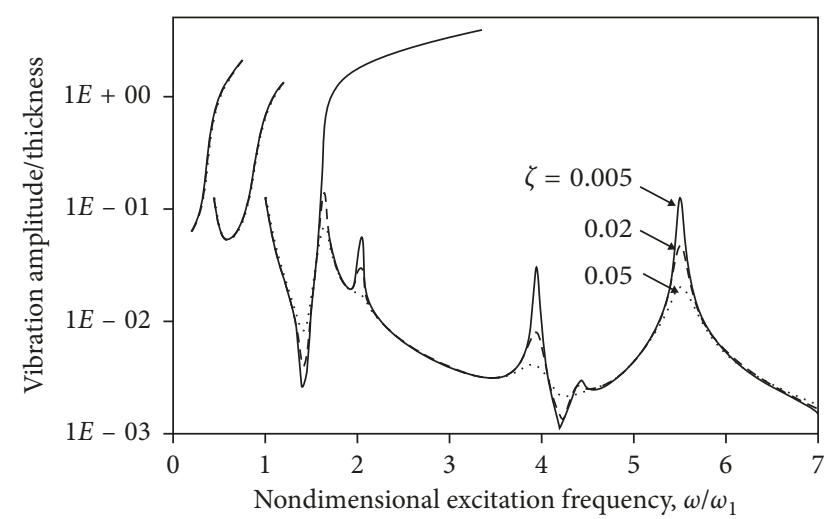

(a)

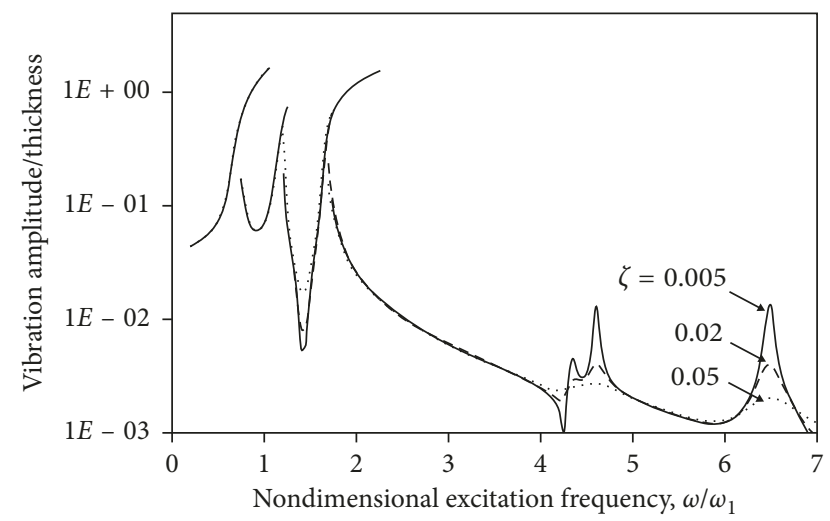

(c)

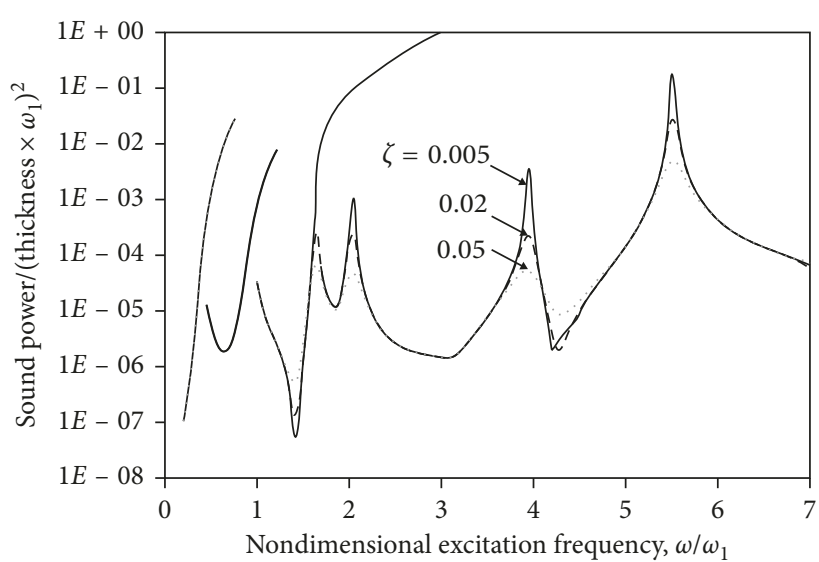

(b)

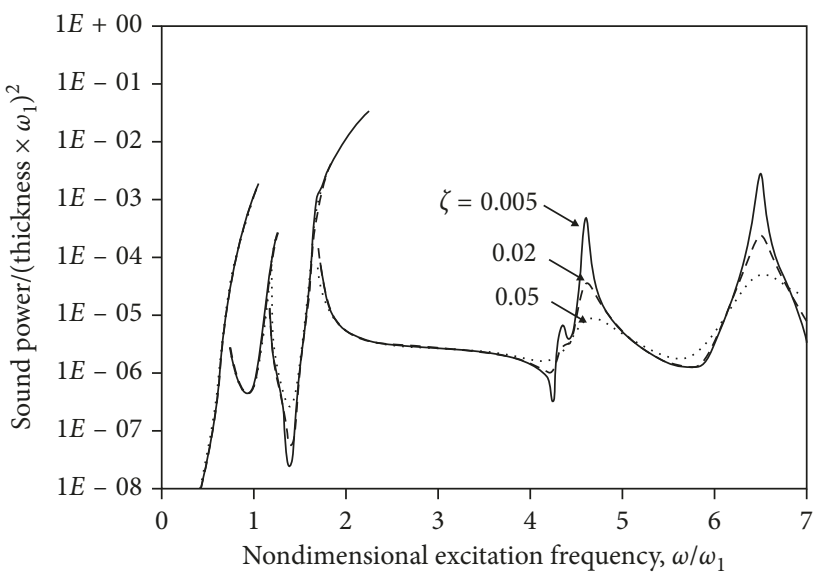

(d)

FIGURE 4: (a) Symmetric and (c) antisymmetric vibration amplitude versus excitation frequency for various damping ratios $(\kappa=0.2, a=3 \mathrm{~m}$, $b=c=0.5 \mathrm{~m}$, and $N=4)$; (b) symmetric and (d) antisymmetric sound radiation versus excitation frequency for various damping ratios $(\kappa=0.2, a=3 \mathrm{~m}, b=c=0.5 \mathrm{~m}$, and $N=4)$.

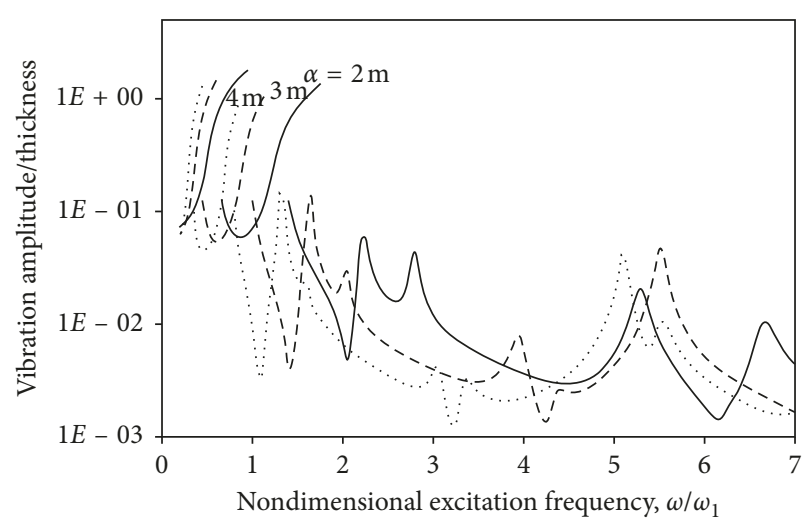

(a)

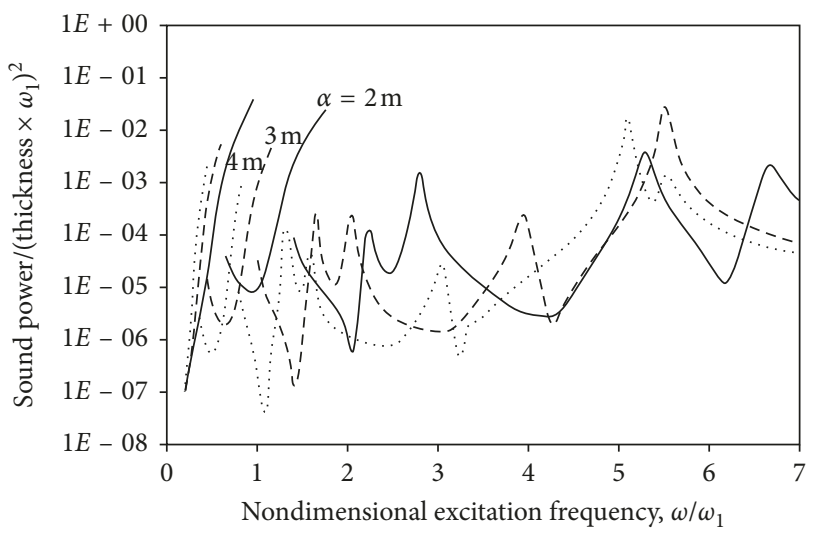

(b)

FIgUre 5: Continued. 


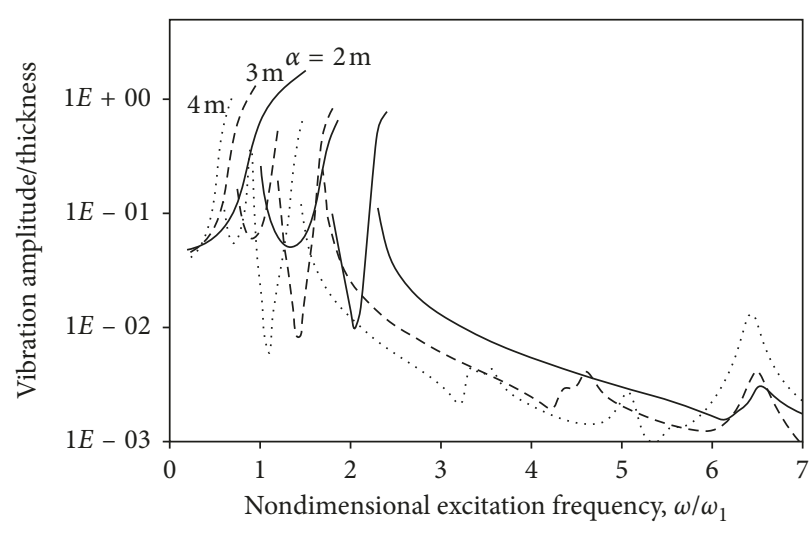

(c)

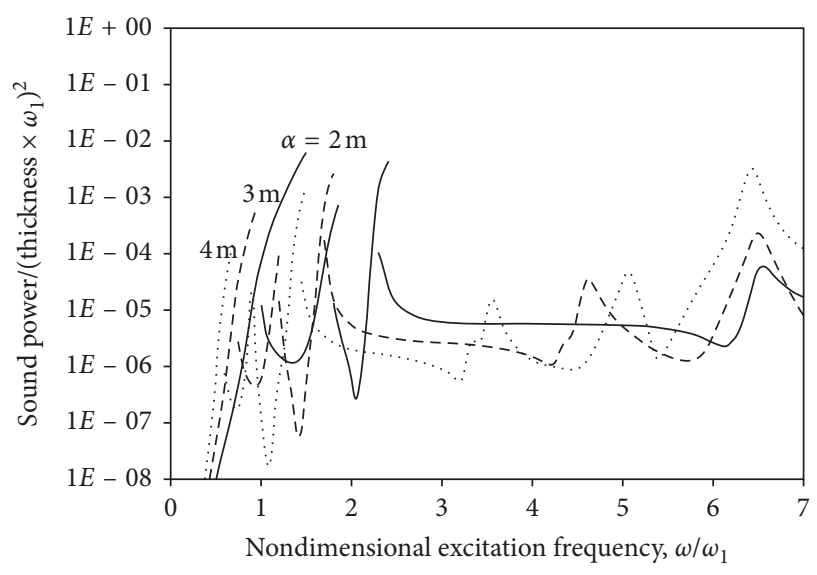

(d)

FiguRE 5: (a) Symmetric and (c) antisymmetric vibration amplitude versus excitation frequency for various panel lengths $(\kappa=0.2$, $b=c=0.5 \mathrm{~m}, \xi=0.02$, and $N=4$ ); (b) symmetric and (d) antisymmetric sound radiation versus excitation frequency for various panel lengths $(\kappa=0.2, b=c=0.5 \mathrm{~m}, \xi=0.02$, and $N=4)$.

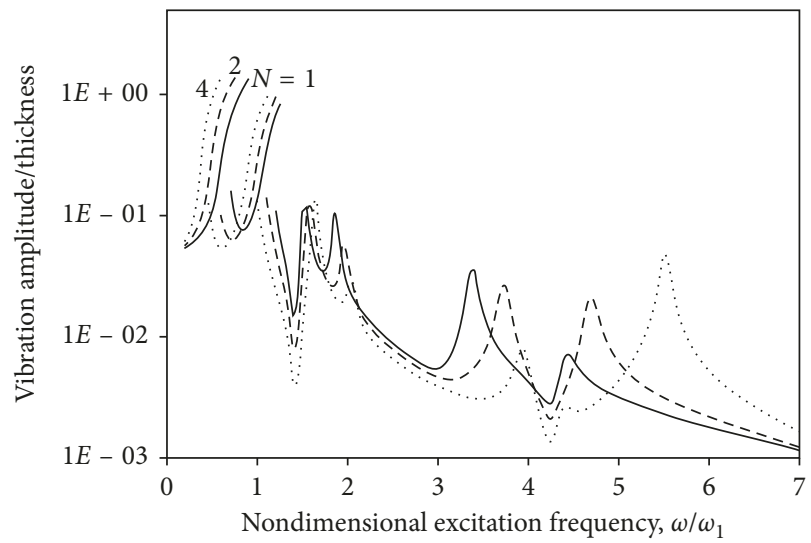

(a)

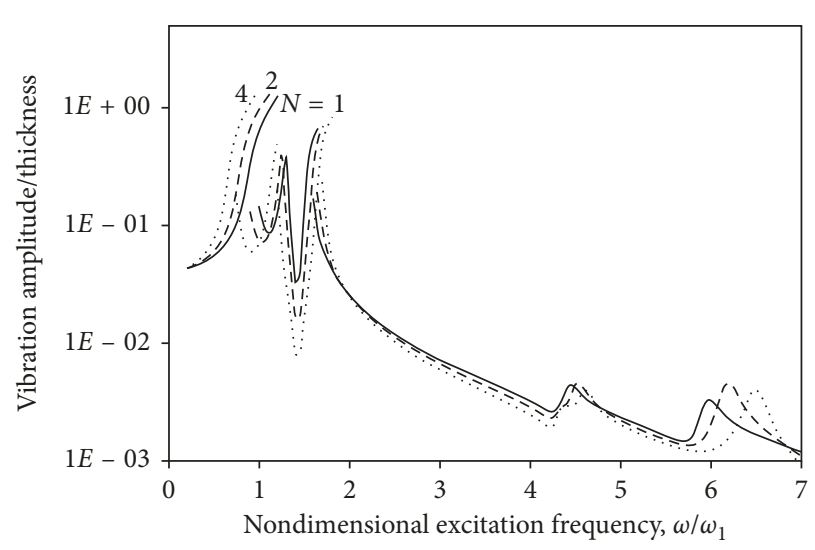

(c)

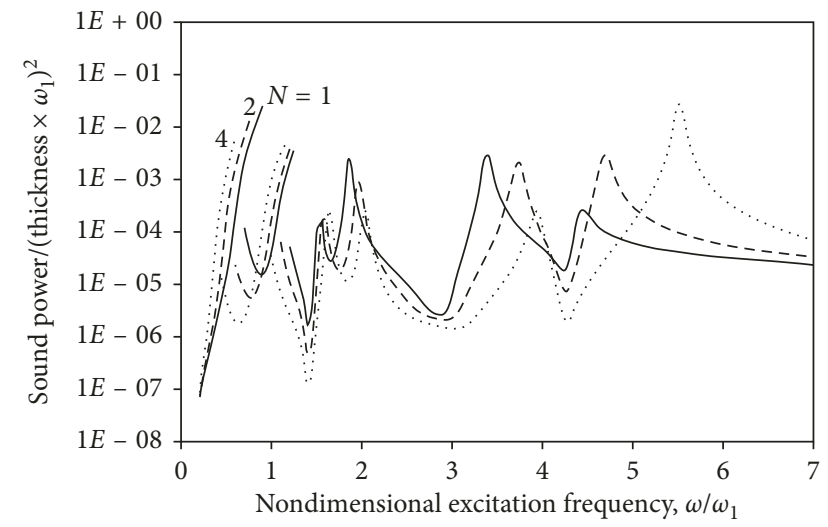

(b)

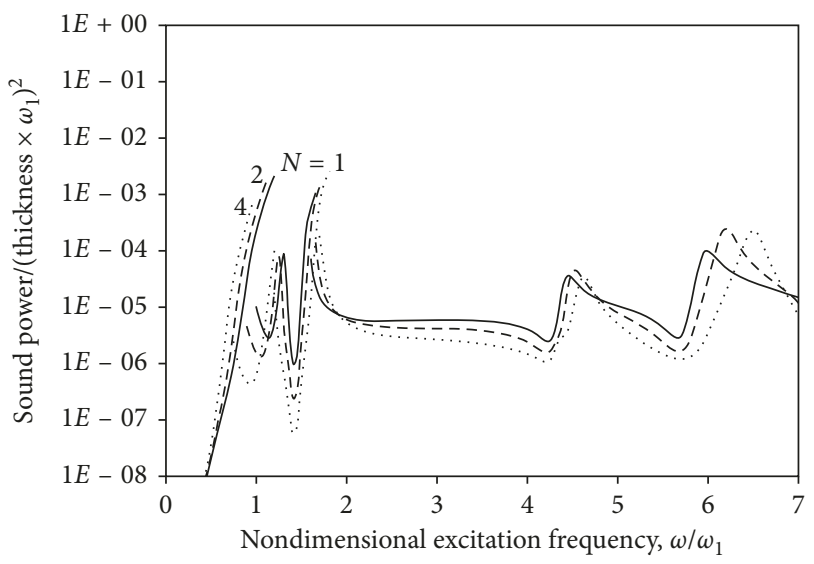

(d)

FIgURE 6: (a) Symmetric and (c) antisymmetric vibration amplitude versus excitation frequency for various numbers of flexible panels $(\kappa=0.2, a=3 \mathrm{~m}, b=c=0.5 \mathrm{~m}$, and $\xi=0.02)$; (b) symmetric and (d) antisymmetric sound radiation versus excitation frequency for various numbers of flexible panels $(\kappa=0.2, a=3 \mathrm{~m}, b=c=0.5 \mathrm{~m}$, and $\xi=0.02)$.

jump in each curve at around $\kappa=0.12$ to 0.15 . The abrupt jumps are due to the jump-up phenomenon, which is well known in forced nonlinear panel vibration [34]. Then, the structural-acoustic peak ratios of vibration amplitude are monotonically decreasing with the increasing excitation level. The structural-acoustic peak ratio of sound power is 


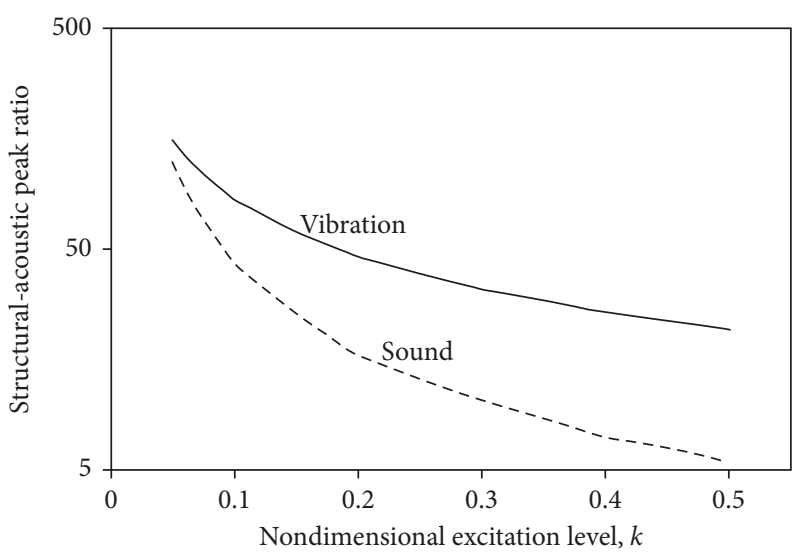

(a)

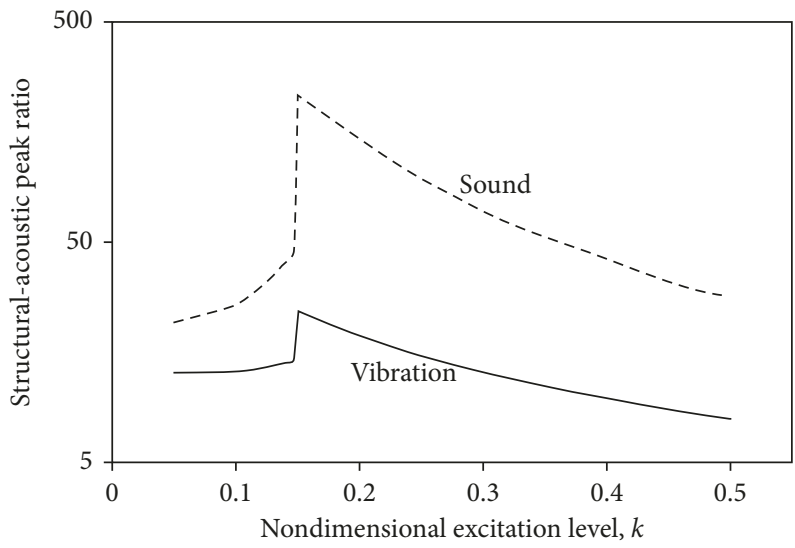

(c)

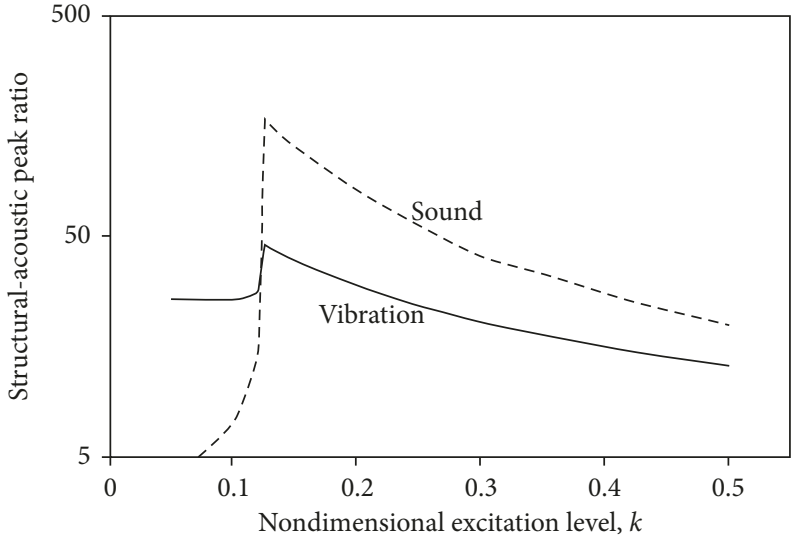

(b)

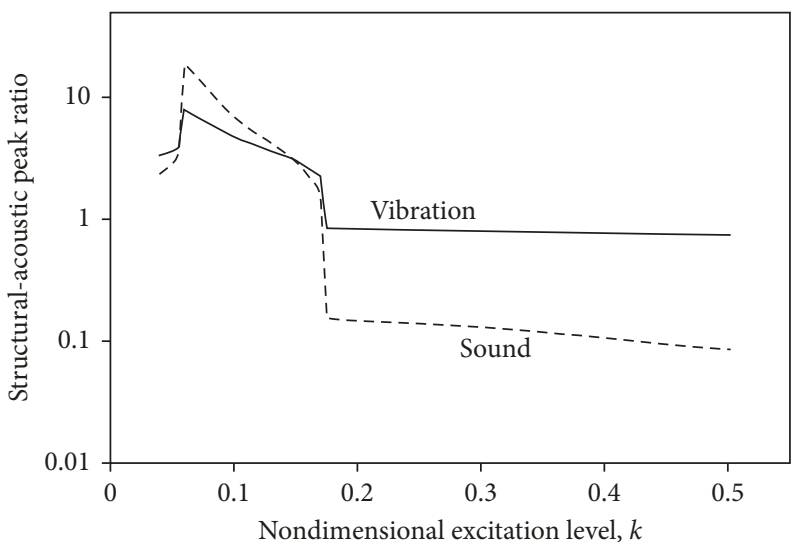

(d)

Figure 7: (a) $(1,1)$ mode, (b) $(2,1)$ mode, (c) $(3,1)$ mode, and (d) $(4,1)$ mode structural-acoustic peak ratios versus excitation level $(a=2$ m, $b=c=0.5 \mathrm{~m}, \xi=0.02$, and $N=4)$.

similar to the other ratios, except it is monotonically increasing when the excitation level is from $\kappa=0.05$ to 0.12 . In Figure $7(\mathrm{~d})$, the two structural-acoustic peak ratios are monotonically increasing for $\kappa=0.05$ to 0.055 . There is an abrupt jump around $\kappa=0.06$. Then, the two ratios are monotonically decreasing until $\kappa=0.17$, at which point another abrupt jump can be seen. The abrupt jumps are also due to the jump-up phenomenon in the acoustic resonance. For $\kappa>0.17$, the structural-acoustic peak ratio of vibration amplitude is almost constant, while the structural-acoustic peak ratio of sound power is mildly decreasing.

\section{Conclusions}

This study investigated the vibration and sound radiation of nonlinear duct panels using the analytic formula based on the classic method combined with Vieta's substitution technique. The main advantage of the analytic formula is that, during the solution procedure, no nonlinear equation solver is required. In other words, the analytic formula is a set of symbolic parameters with various physical meanings. The results obtained from the proposed classic method show reasonable agreement with those from the total harmonic balance method. The main findings can be summarized as follows: (1) the damping ratio and excitation level are the two key factors to determine the nonlinearities of the resonant peaks of nonlinear panels. (2) The acoustic peak values in the sound power figures are generally higher than those in the vibration figures. This implies that the high-frequency components in the sound responses are more significant than those in the vibration responses because the sound radiation efficiency is higher when the response frequency is higher. (3) The antisymmetric modal responses, which have been considered in only a few structural acoustic studies, are found to be as important as (or as large as) the symmetric modal responses. The reason is that the first antisymmetric structural mode is coupled with the first antisymmetric acoustic mode, which is the most dominant acoustic mode even though it is less important than the first symmetric mode. (4) The well-known jump phenomenon is found in the peaks of the structural modes coupled with the acoustic modes, whereas it has previously been found only in the resonant peaks of nonlinear structural vibrations.

\section{Data Availability}

The data used to support the findings of this study are included within the article. 


\section{Conflicts of Interest}

The author declares that there are no conflicts of interest.

\section{Acknowledgments}

The author thanks the financial and technical supports from the City University of Hong Kong. The work described in this paper was fully supported by the CityU SRG (project no. 7004701).

\section{References}

[1] Q. Z. Zhou, D. S. Wang, and Y. Y. Zhu, "An approximate analytical method of the nonlinear vibroacoustic coupling system," Shock and Vibration, vol. 2014, Article ID 315416, 10 pages, 2014.

[2] Y. Y. Lee and E. W. M. Lee, "Widening the sound absorption bandwidths of flexible micro-perforated curved absorbers using structural and acoustic resonances," International Journal of Mechanical Sciences, vol. 49, no. 8, pp. 925-934, 2007.

[3] Y. Y. Lee, R. K. L. Su, C. F. Ng, and C. K. Hui, "The effect of modal energy transfer on the sound radiation and vibration of a curved panel: theory and experiment," Journal of Sound and Vibration, vol. 324, no. 3-5, pp. 1003-1015, 2009.

[4] S. Yang and Q. Yang, "Geometrically nonlinear transient response of laminated plates with nonlinear elastic restraints," Shock and Vibration, vol. 2017, Article ID 2189420, 9 pages, 2017.

[5] J. Y. Fan and J. Huang, "Haar wavelet method for nonlinear vibration of functionally graded CNT-reinforced composite beams resting on nonlinear elastic foundations in thermal environment," Shock and Vibration, vol. 2018, Article ID 9597541, 15 pages, 2018.

[6] Z. Guo, A. Y. T. Leung, and X. Ma, "Solution procedure of residue harmonic balance method and its applications," Science China Physics, Mechanics \& Astronomy, vol. 57, no. 8, pp. 1581-1591, 2014.

[7] A. Y. T. Leung, H. X. Yang, and Z. J. Guo, "Periodic wave solutions of coupled integrable dispersionless equations by residue harmonic balance," Communications in Nonlinear Science and Numerical Simulation, vol. 17, no. 11, pp. 45084514, 2017.

[8] L. Y. Yao, D. J. Yu, X. Y. Cui, and X. G. Zang, "Numerical treatment of acoustic problems with the smoothed finite element method," Applied Acoustics, vol. 71, no. 8, pp. 743-753, 2010.

[9] G. Wang, X. Y. Cui, H. Feng, and G. Y. Li, "A stable nodebased smoothed finite element method for acoustic problems," Computer Methods in Applied Mechanics and Engineering, vol. 297, pp. 348-370, 2015.

[10] J. Huang, K. L. R. Su, Y. Y. R. Lee, and S. Chen, "Various bifurcation phenomena in a nonlinear curved beam subjected to base harmonic excitation," International Journal of Bifurcation and Chaos, vol. 28, no. 7, article 1830023, 2018.

[11] M. Sadri, D. Younesian, and E. Esmailzadeh, "Nonlinear harmonic vibration and stability analysis of a cantilever beam carrying an intermediate lumped mass," Nonlinear Dynamics, vol. 84, no. 3, pp. 1667-1682, 2016.

[12] S. Zhou, G. Song, R. Wang, Z. Ren, and B. Wen, "Nonlinear dynamic analysis for coupled vehicle-bridge vibration system on nonlinear foundation," Mechanical Systems and Signal Processing, vol. 87, pp. 259-278, 2017.
[13] B. Venkatesham, M. Tiwari, and M. L. Munjal, "Prediction of breakout noise from a rectangular duct with compliant walls," International Journal of Acoustics and Vibration, vol. 16, no. 4, pp. 180-190, 2011.

[14] W. C. Tang and W. Z. Lin, "Stiff light composite panels for duct noise reduction," Applied Acoustics, vol. 64, no. 5, pp. 511-524, 2003.

[15] B. Tiseo and A. Concilio, "Passive-active noise control of an acoustic duct," Journal of Theoretical and Applied Mechanics, vol. 49, no. 4, pp. 1135-1149, 2011.

[16] N. Jade and B. Venkatesham, "Experimental study of breakout noise characteristics of flexible rectangular duct," Mechanical Systems and Signal Processing, vol. 108, pp. 156-172, 2018.

[17] D. Younesian, M. Sadri, and E. Esmailzadeh, "Primary and secondary resonance analyses of clamped-clamped microbeams," Nonlinear Dynamics, vol. 76, no. 4, pp. 1867-1884, 2014.

[18] C. K. Hui, Y. Y. Lee, and J. N. Reddy, “Approximate elliptical integral solution for the large amplitude free vibration of a rectangular single mode plate backed by a multi-acoustic mode cavity," Thin-Walled Structures, vol. 49, no. 9, pp. 1191-1194, 2011.

[19] Y. Y. Lee, "Free vibration analysis of a nonlinear panel coupled with extended cavity using the multi-level residue harmonic balance method," Thin-Walled Structures, vol. 98, pp. 332-336, 2016.

[20] Y. Y. Lee, "Structural-acoustic coupling effect on the nonlinear natural frequency of a rectangular box with one flexible plate," Applied Acoustics, vol. 63, no. 11, pp. 1157-1175, 2002.

[21] Y. Qu and G. Meng, "Nonlinear vibro-acoustic analysis of composite sandwich plates with skin-core debondings," AIAA Journal, vol. 55, no. 5, pp. 1723-1733, 2017.

[22] C. K. Hui, Y. Y. Lee, and C. F. Ng, "Use of internally resonant energy transfer from the symmetrical to anti-symmetrical modes of a curved beam isolator for enhancing the isolation performance and reducing the source mass translation vibration: theory and experiment," Mechanical Systems and Signal Processing, vol. 25, no. 4, pp. 1248-1259, 2011.

[23] Z. Aginsky and O. Gottlieb, "Nonlinear fluid-structure interaction of an elastic panel in an acoustically excited twodimensional inviscid compressible fluid," Physics of Fluids, vol. 25, no. 7, article 076104, 2013.

[24] H. T. Banks and R. C. Smith, "Parameter estimation in a structural acoustic system with fully nonlinear coupling conditions," Mathematical and Computer Modelling, vol. 23, no. 4, pp. 17-50, 1996.

[25] L. Xu, "Dynamics of two-strand yarn spinning in forced vibration," Nonlinear Analysis-Theory Methods \& Applications, vol. 71, no. 12, pp. e827-e829, 2009.

[26] L. Xu and L. Sun, "Electromechanical coupled non-linear vibration of the microplate," Proceedings of the Institution of Mechanical Engineers, Part C: Journal of Mechanical Engineering Science, vol. 224, no. 6, pp. 1383-1396, 2010.

[27] Z. L. Huang and X. L. Jin, "Response and stability of a SDOF strongly nonlinear stochastic system with light damping modeled by a fractional derivative," Journal of Sound and Vibration, vol. 319, no. 3-5, pp. 1121-1135, 2009.

[28] K. Khan, M. A. Akbar, and H. Koppelaar, "Study of coupled nonlinear partial differential equations for finding exact analytical solutions," Royal Society Open Science, vol. 2, no. 7, article 140406, 2015.

[29] N. Jacques, E. M. Daya, and M. Potier-Ferry, "Nonlinear vibration of viscoelastic sandwich beams by the harmonic 
balance and finite element methods," Journal of Sound and Vibration, vol. 329, no. 20, pp. 4251-4265, 2010.

[30] S. H. Chen, Y. K. Cheung, and H. X. Xing, "Nonlinear vibration of plane structures by finite element and incremental harmonic balance method," Nonlinear Dynamics, vol. 26, no. 1, pp. 87-104, 2001.

[31] M. K. Lee, M. Hosseini Fouladi, and S. Narayana Namasivayam, "Natural frequencies of thin rectangular plates using homotopy-perturbation method," Applied Mathematical Modelling, vol. 50, pp. 524-543, 2017.

[32] K. Gao, W. Gao, B. Wu, D. Wu, and C. Song, "Nonlinear primary resonance of functionally graded porous cylindrical shells using the method of multiple scales," Thin-Walled Structures, vol. 125, pp. 281-293, 2018.

[33] Y. X. Hao, L. H. Chen, W. Zhang, and J. G. Lei, "Nonlinear oscillations, bifurcations and chaos of functionally graded materials plate," Journal of Sound and Vibration, vol. 312, no. 4-5, pp. 862-892, 2008.

[34] Y. Y. Lee, "Analytic solution for nonlinear multimode beam vibration using a modified harmonic balance approach and Vieta's substitution," Shock and Vibration, vol. 2016, Article ID 3462643, 6 pages, 2016.

[35] A. Shooshtari and S. E. Khadem, "A multiple scales method solution for the free and forced nonlinear transverse vibrations of rectangular plates," Structural Engineering and $\mathrm{Me}$ chanics, vol. 24, no. 5, pp. 543-560, 2006.

[36] Y. Y. Lee, J. L. Huang, C. K. Hui, and C. F. Ng, "Sound absorption of a quadratic and cubic nonlinearly vibrating curved panel absorber," Applied Mathematical Modelling, vol. 36, no. 11, pp. 5574-5588, 2012.

[37] Y.-Y. Lee, "The effect of leakage on the sound absorption of a nonlinear perforated panel backed by a cavity," International Journal of Mechanical Sciences, vol. 107, pp. 242-252, 2016.

[38] H. N. Chu and G. Herrmann, "Influence of large amplitudes on free flexural vibrations of rectangular elastic plates," Journal of Applied Mechanics, vol. 23, pp. 523-540, 1956.

[39] C. Y. Chia, Nonlinear Analysis of Plates, McGraw-Hill Inc., New York City, NY, USA, 1980.

[40] S. P. Timoshenko and J. N. Goodier, Theory of Elasticity, McGraw-Hill Inc., New York City, NY, USA, 3rd edition, 1970.

[41] F. Fahy, Sound and Structural Vibration, Radiation, Transmission and Response, Academic Press, Cambridge, MA, USA, 6th edition, 2000. 


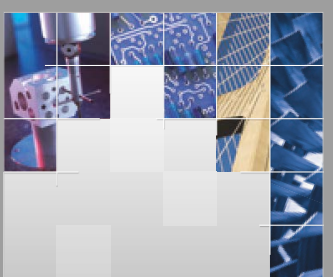

\section{Enfincering}
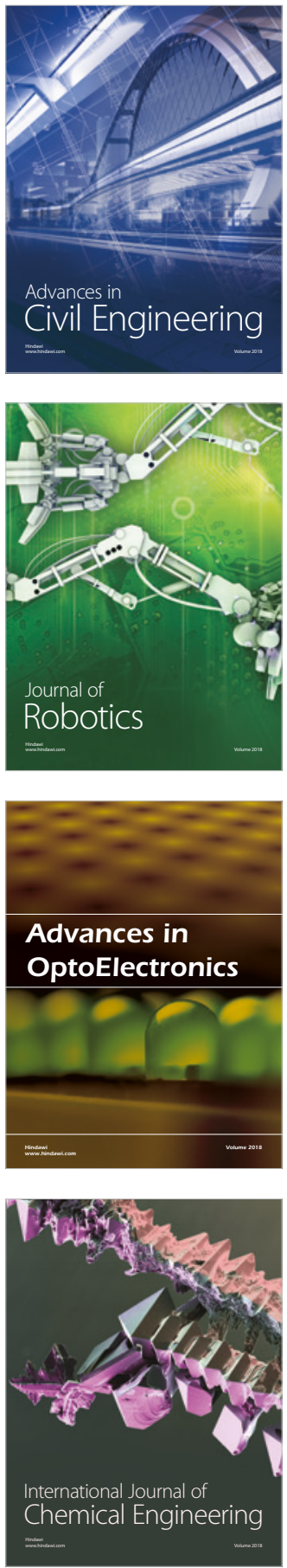

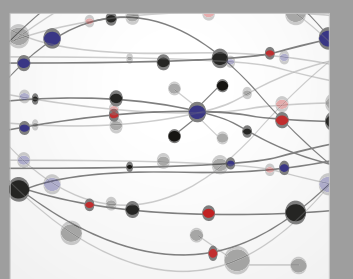

\section{Rotating \\ Machinery}

The Scientific World Journal

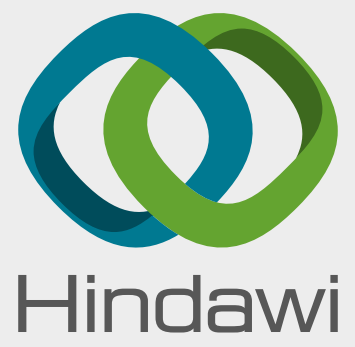

Submit your manuscripts at

www.hindawi.com
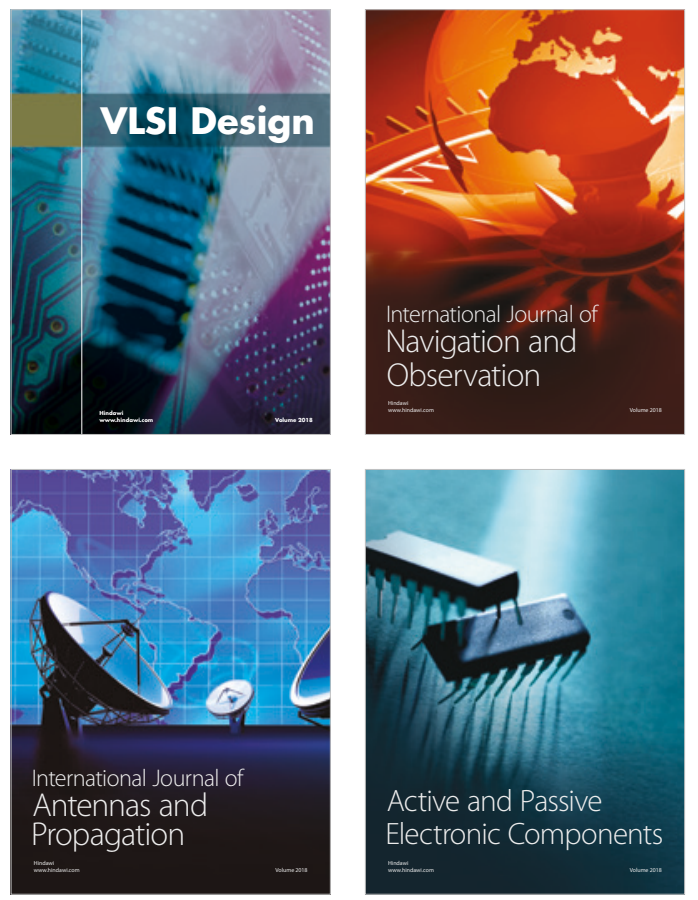
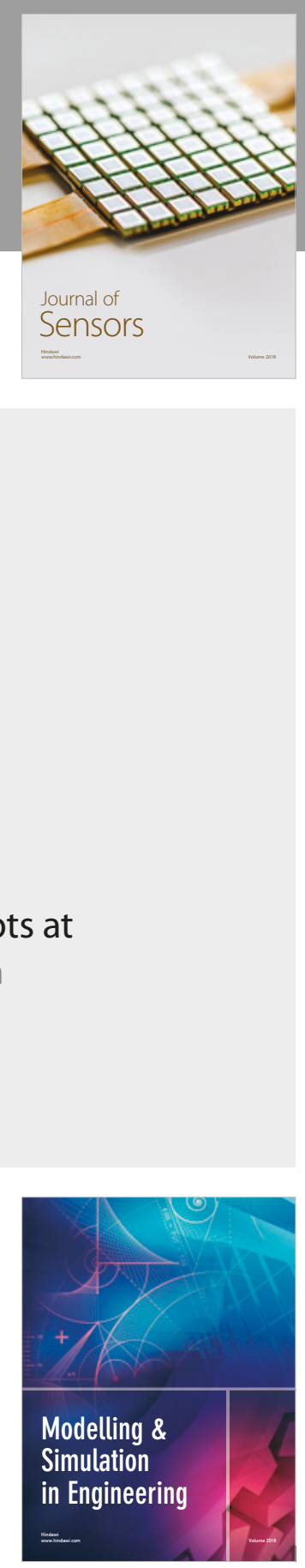

\section{Advances \\ Multimedia}
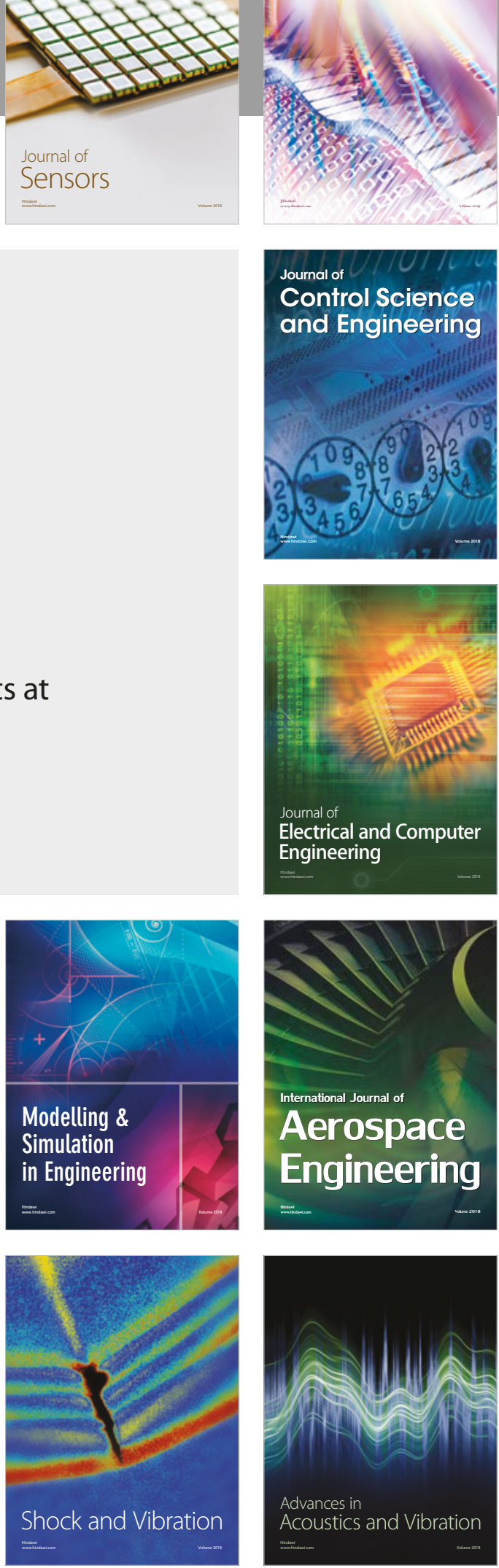